\title{
Delta doping and positioning effects of type II GaSb quantum dots in GaAs solar cell
}

\author{
Juanita Saroj James ${ }^{\mathrm{a}}$, Hiromi Fujita ${ }^{\mathrm{b}}$, N. Fernández-Delgado ${ }^{\mathrm{c}}$, M. Herrera ${ }^{\mathrm{c}}$, S. I. Molina ${ }^{\mathrm{c}}$,
} Andrew R.J. Marshall ${ }^{\mathrm{a}}$, Anthony Krier ${ }^{\mathrm{a}}$

${ }^{a}$ Physics Department, Lancaster University, Lancaster, LA1 4YB, UK

${ }^{\mathrm{b}}$ Magnetic Sensors Process Technology \& Development Department, Asahi-Kasei

Microdevices, Japan

${ }^{\mathrm{c}}$ Instituto de Microscopía Electrónica y Materiales, Departamento de Ciencia de los

Materiales e I.M. y Q.I., Facultad de Ciencias, 11510-Puerto Real, Cádiz (Spain)

\begin{abstract}
GaSb quantum dot (QD) solar cell structures were grown by molecular beam epitaxy on GaAs substrates. We investigate the reduction in open circuit voltage and study the influence of the location of QD layers and their delta doping within the solar cell. Devices with 5 layers of delta doped QDs placed in the intrinsic, $\mathrm{n}$ and $\mathrm{p}$ regions of a GaAs solar cell are experimentally investigated and the deduced values of $J_{\mathrm{sc}}, \mathrm{V}_{\mathrm{oc}}$, Fill factor, efficiency $(\eta)$ are compared. A trade-off is needed to minimize the $\mathrm{V}_{\text {oc }}$ degradation while maximizing the short circuit current density $\left(\mathrm{J}_{\mathrm{sc}}\right)$ enhancement due to sub-bandgap absorption. The voltage recovery is attributed to the removal of the QDs from the high field region which reduces SRH recombination. The devices with $\mathrm{p}$ or $\mathrm{n}$ doped QDs placed in the flat band potential ( $\mathrm{p}$ or $\mathrm{n}$ region) show a recovery in $\mathrm{J}_{\mathrm{sc}}$ and $\mathrm{V}_{\mathrm{oc}}$ compared to devices with delta doped QDs placed in the depletion region. However there is less photocurrent arising from the absorption of subband gap photons. Furthermore, the long wavelength photoresponse of the $\mathrm{n}$ doped QDs placed in the $\mathrm{n}$ region shows a slight improvement compared to the control cell. The approach
\end{abstract}


of placing QDs in the $\mathrm{n}$ region of the solar cell instead of the depletion region is a possible route towards increasing the conversion efficiency of QD solar cells.

\section{Keywords}

Solar cells, Quantum dots, Molecular beam epitaxy, Gallium Antimonide, Open circuit voltage $\left(\mathrm{V}_{\mathrm{oc}}\right)$, Photocurrent, delta doping, Photoresponse

\section{Introduction}

The use of nanostructured materials in solar cells enables one to tune their absorption properties leading to a better match to the solar spectrum and subsequently an increased photocurrent. Type II GaSb/GaAs quantum dots (QDs) can significantly extend the spectral response beyond the visible out towards $1.4 \mu \mathrm{m}$ giving a near optimum band gap for concentrator solar cell applications[1]. However, the introduction of GaSb QDs in GaAs solar cells degrades the open circuit voltage $\left(\mathrm{V}_{\mathrm{oc}}\right)$ and hence lowers the overall efficiency of the device. Experiments have shown that QDs embedded in the depletion region could generate additional photocurrent. But, electron-hole recombination in QDs results in additional dark current which reduces the open circuit voltage and keeps the conversion efficiency of QD solar cells below the Shockley-Queisser limit. Using a GaSb/GaAs type II QD absorber embedded in the p-doped region of an ideal solar cell but spatially separated from the depletion region is expected to lead to voltage preservation[2]. Meanwhile, improvements in voltage preservation through suppression of hole thermionic emission rates by n-type doping at the expense of reduction in short circuit current and by positioning of QD layers at the edge of the space charge region have been reported[3]. Previous studies have also shown that delta doping of quantum dots flattens the band structure and reduces SRH recombination and dark current leading to voltage preservation[4]. Hence, it is important to design the quantum dot 
solar cell by considering two major factors i.e. position of the quantum dots within the solar cell[5] and their doping profile[6]. The purpose of this study is to investigate the approach of placing QDs in the $\mathrm{n}$ or $\mathrm{p}$ region of the solar cell and the effects of delta doping of quantum dots.

\section{Experiment}

The GaSb QD solar cell structures were grown by molecular beam epitaxy on GaAs substrates as shown in figure 1. The structures contain 5 layers of delta doped QDs embedded in the intrinsic (sample B and C), $\mathrm{n}$ doped (sample D) and p doped (sample E) regions of the GaAs p-i-n junction, where the QDs in D and E are spatially separated from the depletion region. The QDs were grown using the Stranski-Krastanow self-assembled growth method following our previous work[1]. First, a $1 \mu \mathrm{m}$ thick n-type GaAs: Te base layer with a doping density of $10^{17} \mathrm{~cm}^{-3}$ was grown, followed by a $500 \mathrm{~nm}$ intrinsic region. Then a $0.5 \mu \mathrm{m}$-type GaAs: Be $\left(2 \times 10^{18} \mathrm{~cm}^{-3}\right)$ emitter layer, a $50 \mathrm{~nm} \mathrm{Al}_{0.8} \mathrm{Ga}_{0.2} \mathrm{As}$ window layer and finally a thin $50 \mathrm{~nm}$ GaAs cap were grown. The GaSb QDs were separated by $35 \mathrm{~nm}$ GaAs barriers. Figure 2 shows the high angle annular dark field (HAADF) scanning transmission electron microscopy (STEM) image of a 5 layer stack of GaSb/GaAs QDs. The QD nanostructures are

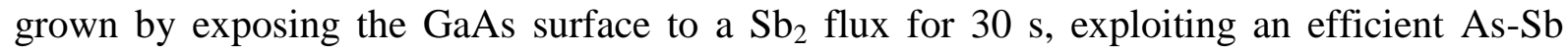
exchange reaction to form a thin $\sim 0.5$ monolayer (ML) GaSb layer. The Ga and the Sb cells are then opened simultaneously and 2.1 ML of GaSb is deposited. The electron diffraction pattern was used to monitor the growth and was observed to change from streaky to spotty after depositing 1.5 ML of GaSb. Figure 3 shows the location of the doped/Undoped GaSb QDs at the different regions within the solar cell. The solar cells were fabricated from the epitaxial material as $2.5 \mathrm{~mm}$ diameter mesa etched diodes by using standard photolithography and wet etching techniques. None of the solar cells were provided with an anti-reflection 
coating. Current -voltage (I-V) curves were taken with a Keithley SMU at room temperature and under 1 sun illumination provided by an LOT Oriel solar simulator. The external quantum efficiency (EQE) was measured using a 100 W tungsten halogen light source, monochromator, lock-in-amplifier and a calibrated $\mathrm{Si} / \mathrm{InGaAs}$ detector.

\section{Results and discussion}

Figure 4 shows the I-V characteristics and external quantum efficiency of the quantum dot solar cells (QDSCs) and the GaAs reference cell. The deduced values of $\mathrm{J}_{\mathrm{sc}}, \mathrm{V}_{\mathrm{oc}}$, Fill factor and conversion efficiency are listed in Table 1 . To begin with we consider the effect of delta doping of QDs placed in the intrinsic region on the performance of the solar cell. The $\mathrm{V}_{\text {oc }}$ of the QD solar cell without delta doping (sample A) is $0.62 \mathrm{~V}$, which is much lower than the GaAs reference sample $R$. The reduction in the $V_{o c}$ of sample $A$ in the intrinsic region is due to the accumulation of holes trapped within the quantum dots. The Photo-generated minority holes from the base region undergo drift across the depletion region and are captured by the QDs thereby reducing the short-circuit current. These trapped holes then act as recombination centres, decreasing the open-circuit voltage.

Improvements in $\mathrm{V}_{\mathrm{oc}}$ are observed for $\mathrm{n}$ - doping of QDs (sample $\mathrm{B}$ ) whereas the p-doping (sample C) further degraded the solar cell performance, i.e. both $\mathrm{V}_{\mathrm{oc}}$ and $\mathrm{J}_{\mathrm{sc}}$ are reduced (fig 4).The purpose of modulated n- doping is to partially fill the quantum dots with electrons to reduce recombination which helps to recover the $\mathrm{V}_{\mathrm{oc}}$. But the transition probability of electrons from the valence band to conduction band is reduced in the QDs which weakens the absorption at the long wavelength range. The device with p-doped QDs placed in the intrinsic region shows the worst performance. As the intentionally doped holes are strongly localized in QDs more holes get accumulated in the QDs and recombine with electrons leading to the 
reduction of $\mathrm{V}_{\mathrm{oc}}$. Although the photocurrent of the cell is reduced, the sub-bandgap photoresponse is greatly increased. It is interesting that a strong extended photoresponse is observed for p-doped quantum dots in the intrinsic region (Sample B), higher than that of the undoped solar cell (Sample A).This is because the transition probability of an electron from the valence band to the conduction band via the QD hole states (which could be considered as an intermediate band) is greatly increased. The photocurrent contributions by the sub-band gap photons can be calculated by integrating the product of $\mathrm{EQE}$ and spectral irradiance of the tungsten halogen light source (see Table 1). Thus the contribution from the p doped QDs and sub-band gap photons to the total short circuit current (as shown in table 1) is approximately equal to $0.85 \%$ which is higher than in the other devices studied here.

Considering the degradation of the extended spectral response from the QDSC in sample B and the conversion efficiency of sample $\mathrm{C}$, a trade-off is needed; i.e. to minimize the $\mathrm{V}_{\mathrm{oc}}$ degradation while maximizing the short circuit current density $\left(\mathrm{J}_{\mathrm{sc}}\right)$ due to sub-bandgap absorption. To achieve this, both the location and the doping of the QD layers needs to be optimised and therefore we studied the influence of the position of delta doped QDs in the $n$ or $\mathrm{p}$ regions of the solar cell. Figure 5 shows the I-V characteristics of $n$ and $p$ delta doped QDs placed in the $\mathrm{n}$ or $\mathrm{p}$ regions of the solar cell. In both cases the $\mathrm{V}_{\mathrm{oc}}$ of the delta doped QDs placed in $\mathrm{n}$ and $\mathrm{p}$ regions of the solar cells shows a significant recovery. The $\mathrm{J}_{\mathrm{sc}}$ and $\mathrm{V}_{\mathrm{oc}}$ of Sample D (n-QDs in n-region) and Sample E (p-QDs in p-region) are greater than Sample B (n-QDs in i-region) and sample C (p-QDs in i-region) because the QDs are located in the flat band region away from the depletion region. A slight increase in the long wavelength photoresponse is attributed to the increased electron population in the QD from the $n$ - $\delta$-doping which enables sub band gap absorption. But, this is limited due to the electrons around the QDs on the n-side. 
The device with p-doped QDs placed in the flat band potential region (sample E) shows the highest conversion efficiency compared to other QD devices studied here. However, there is almost no contribution to the sub-band gap photocurrent from the QDs. This behaviour is in contrast to sample C (p QDs in i-region) which gave the highest photoresponse. Hence, the pdoping of QDs placed in the i or p region does not favour the overall solar cell performance.

It has been previously shown that the $\mathrm{n}$ delta doping of QDs increases the low band gap absorption and also increases the conversion efficiency[4][7]. Our studies show the importance of positioning and doping of the QDs within the solar cell structure. In the device with n-delta doped QDs placed in the $\mathrm{n}$ region, the $\mathrm{V}_{\mathrm{oc}}$ and $\mathrm{J}_{\mathrm{sc}}$ is not fully recovered compared to the control cell. But, our results show that the device with n-doped QDs placed in the nregion has a higher open circuit voltage compared with n-doped QDs placed in the depletion region. The sub bandgap photocurrent is also higher and could be further increased by stacking additional layers of QDs without compromising the open circuit voltage. The conversion efficiency could be further improved by finding an optimum doping level.

\section{Conclusions}

In summary, we presented the effects of delta doping and positioning of GaSb QDs on the performance of GaAs single junction solar cells in terms of current-voltage characteristics and photoresponse. The $\mathrm{V}_{\mathrm{oc}}$ for the solar cell with delta doped QDs located in the $\mathrm{p}$ or $\mathrm{n}$ region is recovered compared to that for undoped QDs in the intrinsic region. This voltage recovery is attributed to the position of the QDs away from an area of highest electric field and reduced SRH recombination. The approach of placing QDs in the n-region of the solar cell instead of the depletion region may be useful in helping to increase the conversion efficiency of solar cells because the long wavelength response could be further improved by stacking more layers of QDs. 


\section{Acknowledgements}

This work was supported by EU Marie-Curie Training networks PROPHET (PITN-GA-2010264687) and PROMIS (H2020-MSCA-ITN-2014-641899). H. Fujita would like to thank Asahi Kasei Corporation for the award of an industry scholarship. A.R.J. Marshall gratefully acknowledges support of a fellowship from the Royal Academy of Engineering.

[1] Peter James Carrington, Abu Syed Mahajumi, Magnus C. Wagener, Johannes Reinhardt Botha, Qian Zhuang, Anthony Krier. 'Type II GaSb/GaAs quantum dot/ring stacks with extended photoresponse for efficient solar cells', Physica B 407 (2012), 1493-1496

[2] A. Kechiantz, A. Afanasev, J.-L. Lazzari. 'Impact of Spatial Separation of Type-II GaSb Quantum Dots from the Depletion Region on the Conversion Efficiency Limit of GaAs Solar Cells', Mesoscale and Nanoscale physics, arXiv:1310.5075

[3] J. Hwang, A. J. Martin, K. Lee, S. Forrest, J. Millunchick, and J. Phillips. 'Preserving Voltage and Long Wavelength Photoresponse in GaSb/GaAs Quantum Dot Solar Cells', 39th IEEE Photovoltaic Specialists Conference (Tampa, FL, 2013).

[4] Stephen J Polly, David V Forbes, Kristina M Driscoll, Seth M Hubbard 'Delta Doping Effects on Quantum Dot Solar Cells', IEEE journal of Photovoltaics Vol.4,No.4.

[5] D. Zhou, P.E. Vullum, G. Sharma, S.F. Thomassen, R. Holmestad, T.W. Reenaas, B.O. Fimland. 'Positioning effects on quantum dot solar cells grown by molecular beam epitaxy', Appl. Phys. Lett., 96 (2010), p. 83108

[6] Yeongho Kim, Keun-Yong Ban, Darius Kuciauskas, Patricia C Dippo and Christiana B Honsberg. 'Impact of delta-doping position on photoluminescence in type-II InAs/GaAsSb quantum dots',Semicond. Sci. Technol. 30 (2015) 035006 (6pp)

[7] K. Sablon, J. Little, V. Mitin, A. Sergeev, N. Vagidov, and K. Reinhardt, 'Strong Enhancement of Solar Cell Efficiency Due to Quantum Dots with Built-In Charge', N anoLett., 11, 2011, pp. , 2311-2317.

[8] P. J. Carrington, M. C. Wagener, J. R. Botha, A. M. Sanchez, A. Krier. 'Enhanced infrared photo-response from GaSb/GaAs quantum ring solar cells', Applied Physics Letters 12/2012, 101(23). 
Figures and Captions

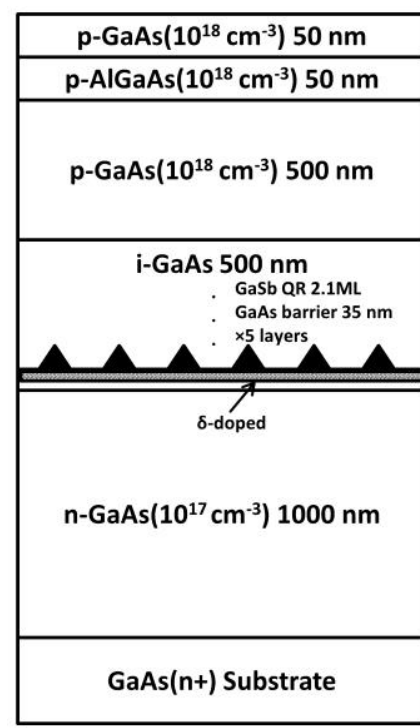

Sample A - Undoped Sample B- $\mathbf{n}$ doped

Sample C-p doped

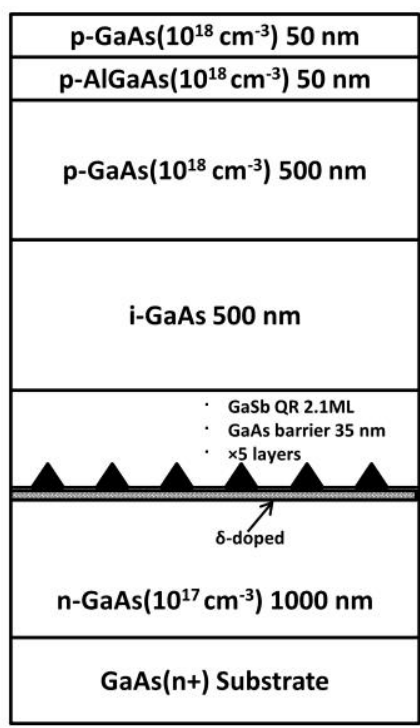

Sample D

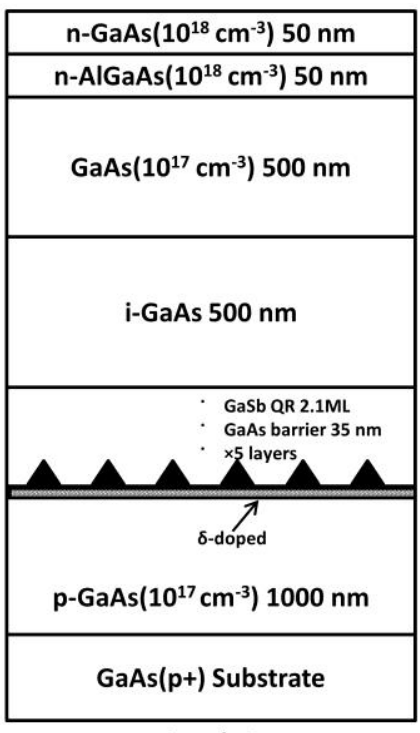

Sample E

Figure 1: Schematic structure of QD SCs: Sample A-undoped QDs grown in intrinsic region, Sample B- n-doped QDs grown in intrinsic region, Sample C-p-doped QDs grown in intrinsic region, Sample D-n doped QDs grown in n-region, Sample E-p- doped QDs grown in p-region

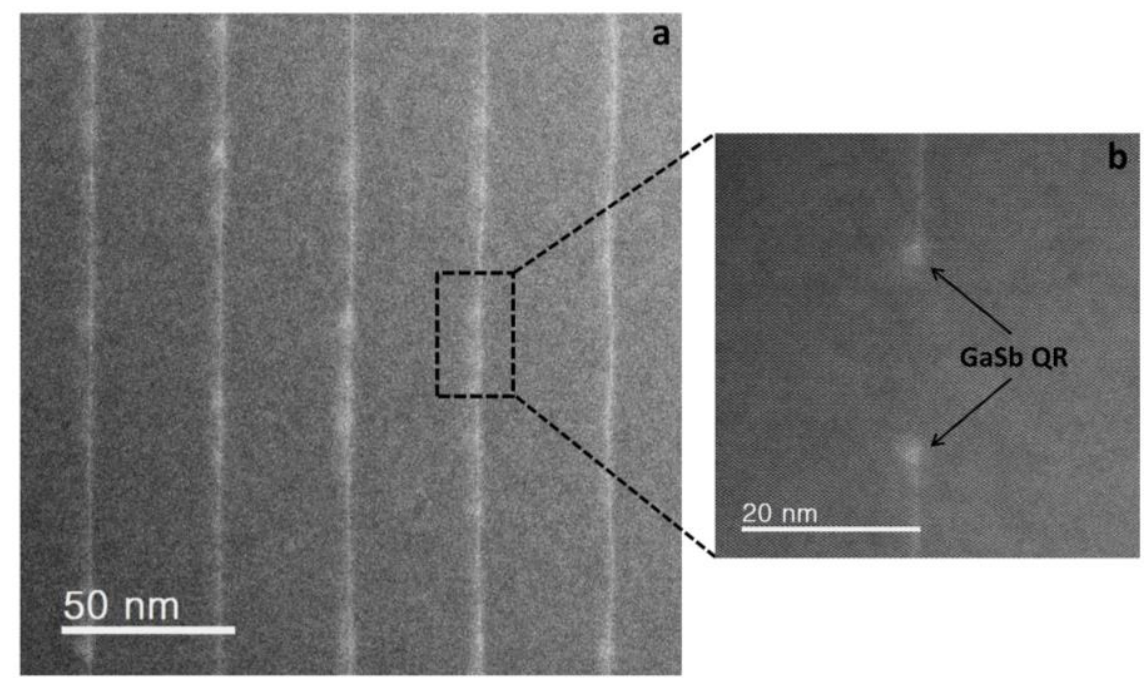

Figure 2: High angle annular dark field (HAADF) scanning transmission electron microscopy (STEM) images (a) 5 layer of GaSb/GaAs QDs with no threading dislocation. (b) Enlarged view of a GaSb quantum ring (QR) 


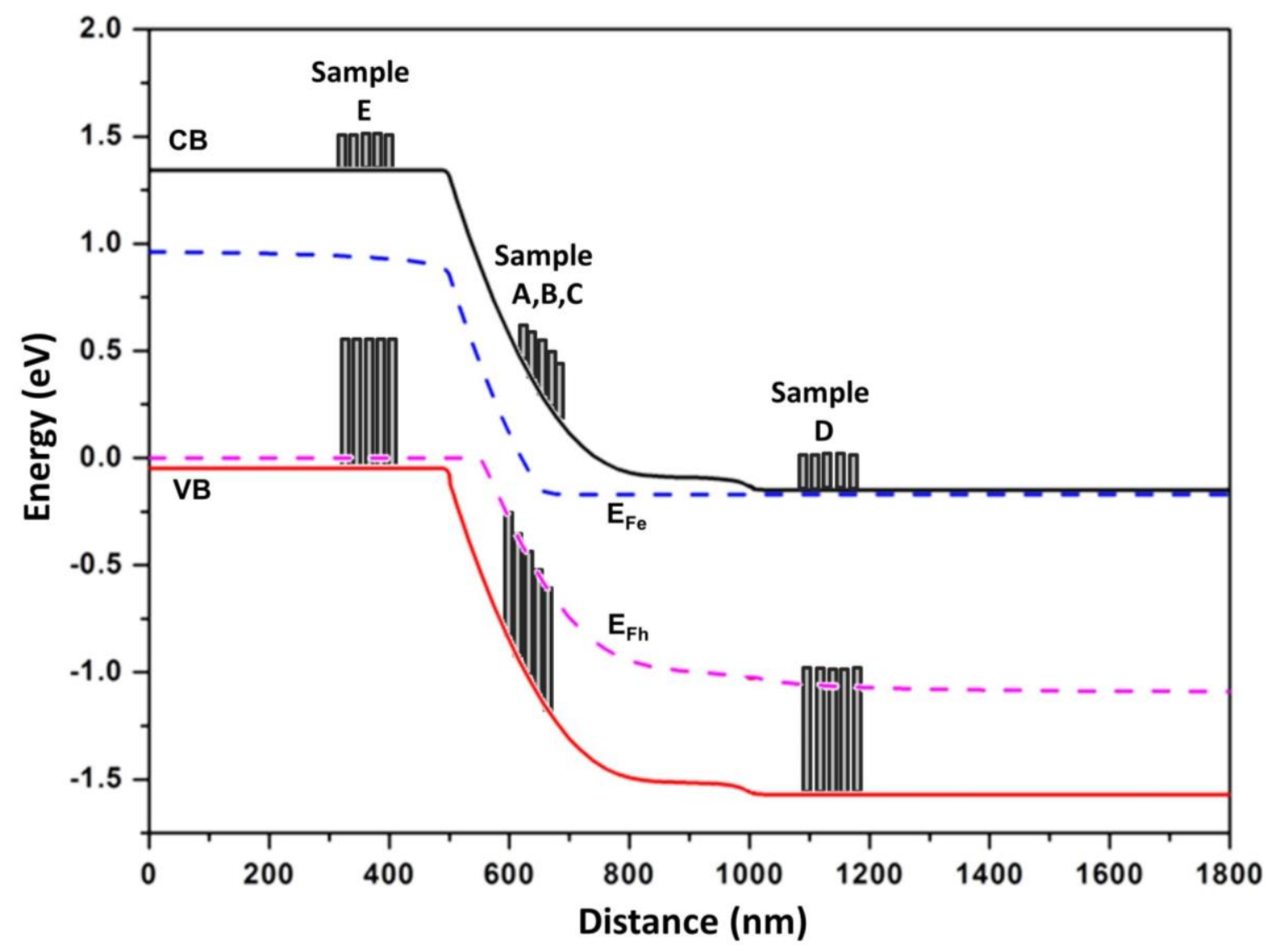

Figure 3: Energy band diagram showing the location of the doped/undoped GaSb QDs at the different locations within the solar cell (band diagram shows the situation at short circuit illumination). 

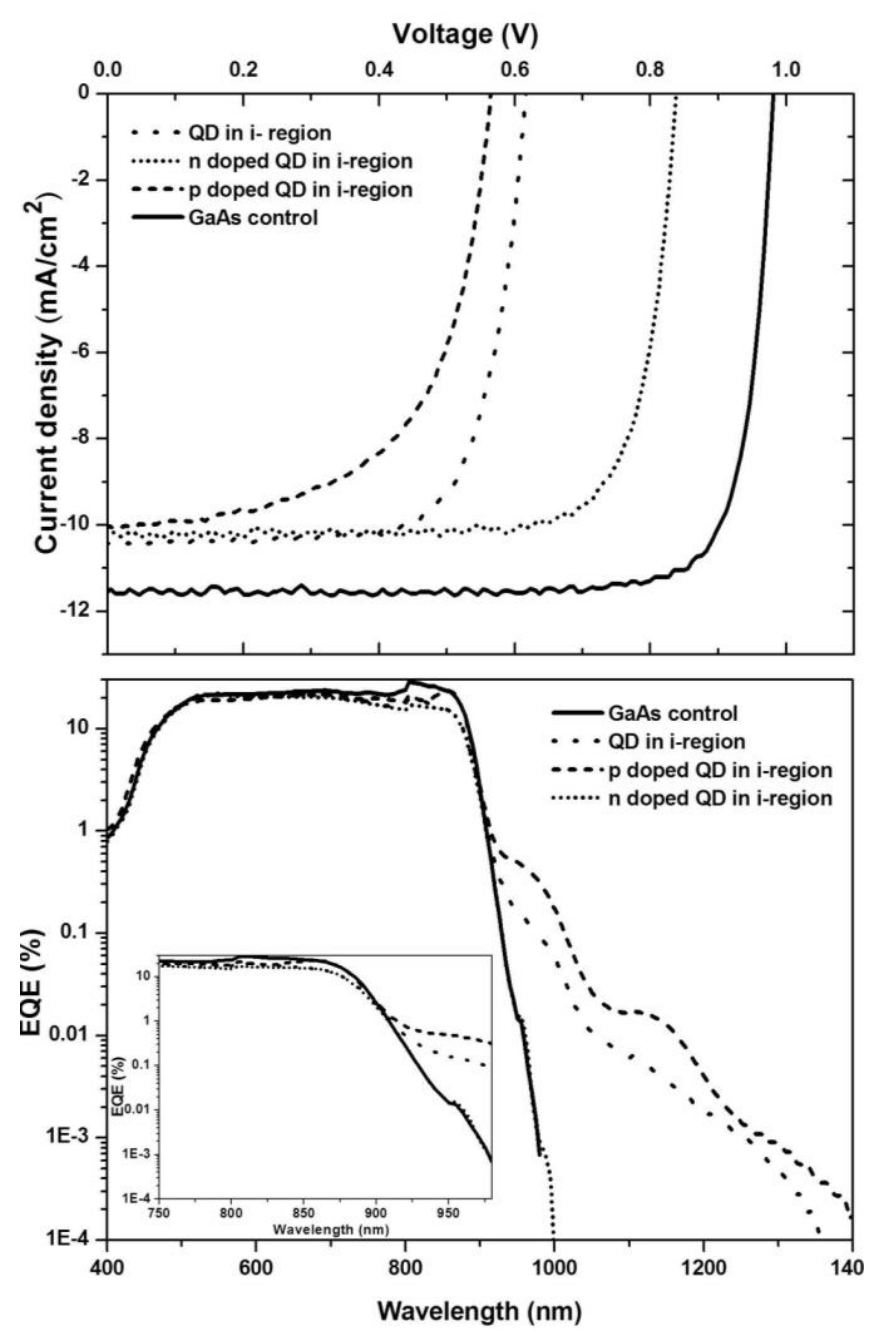

Figure 4: a) I-V characteristics of delta doped QDSCs in i-region b) EQE of delta doped QDSCs in i-region. The inset shows the detailed view of EQE between $800 \mathrm{~nm}$ and 950 nm.

\begin{tabular}{|c|c|c|c|c|c|c|}
\hline & $\begin{array}{c}\text { GaAs } \\
\text { Reference } \\
\mathrm{R}\end{array}$ & $\begin{array}{c}\text { iQDs in } \\
\text { i-region } \\
\mathrm{A}\end{array}$ & $\begin{array}{c}\text { nQDs in } \\
\text { i-region } \\
\text { B }\end{array}$ & $\begin{array}{c}\text { pQDs in } \\
\text { i-region } \\
\text { C }\end{array}$ & $\begin{array}{c}\text { nQDs in } \\
\text { n-region } \\
\text { D }\end{array}$ & $\begin{array}{c}\text { pQDs in } \\
\text { p-region } \\
\text { E }\end{array}$ \\
\hline $\mathrm{J}_{\mathrm{sc}}\left(\mathrm{mA} / \mathrm{cm}^{2}\right)$ & 11.5 & 10.4 & 10.2 & 10.04 & 10.9 & 11.2 \\
\hline $\mathrm{V}_{\text {oc }}(\mathrm{V})$ & 0.98 & 0.62 & 0.84 & 0.56 & 0.86 & 0.89 \\
\hline Fill factor $(\%)$ & 82 & 72 & 78 & 59 & 76 & 80 \\
\hline Efficiency $(\%)$ & 9.2 & 4.6 & 6.6 & 3.3 & 7.1 & 8.0 \\
\hline $\begin{array}{c}\text { Integrated } \mathrm{J}_{\mathrm{sc}} \text { from } \mathrm{EQE} \\
\text { above } 930 \mathrm{~nm}\left(\mu \mathrm{A} / \mathrm{cm}^{2}\right)\end{array}$ & 1.5 & 29 & 1.7 & 85 & 3.9 & 0.75 \\
\hline
\end{tabular}

Table 1: Comparison of Quantum dot solar cells to GaAs reference solar cells 

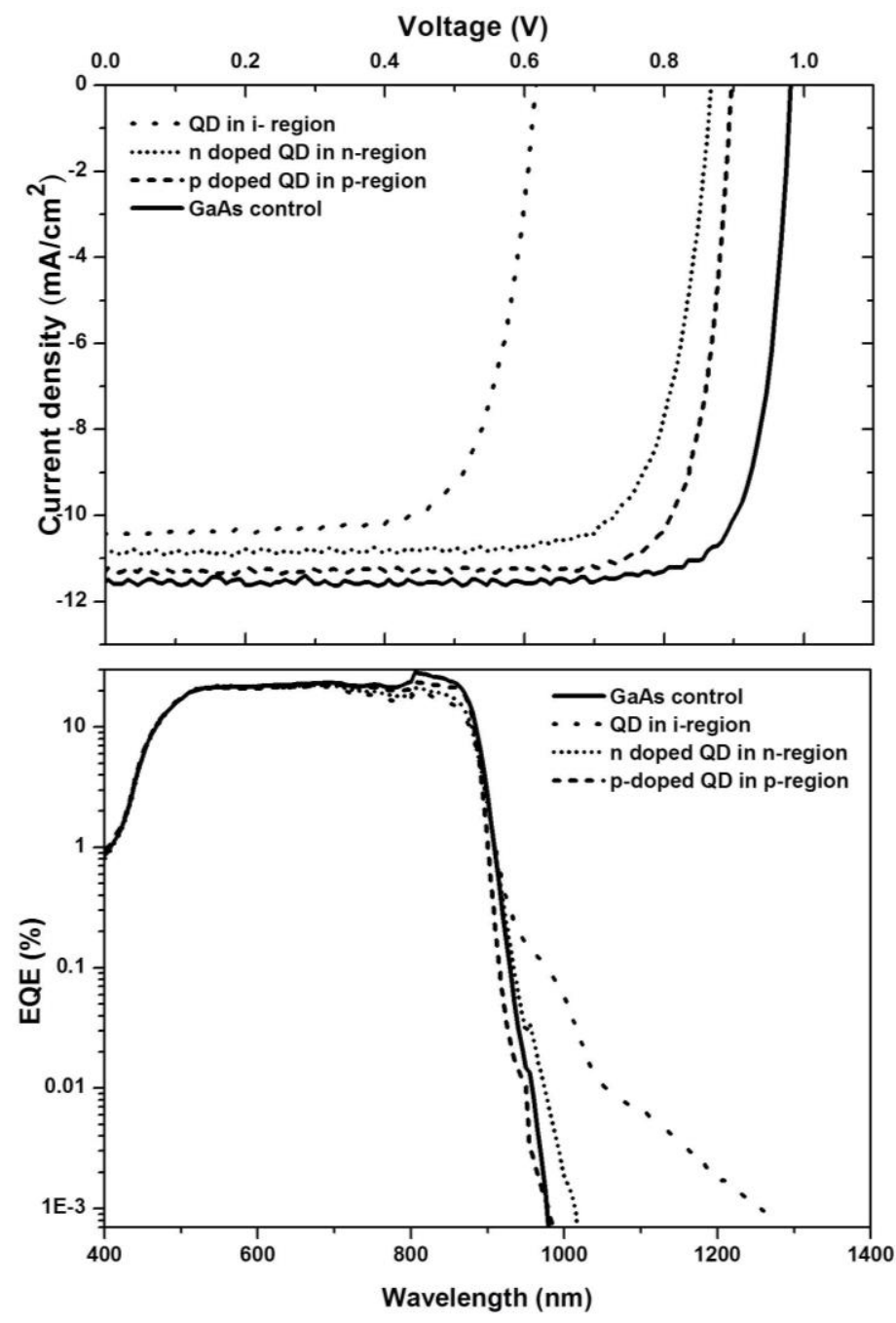

Figure 5: a) I-V characteristics of delta doped QDSCs in n or p-region b) EQE of delta doped QDSCs in n or p-region 